\title{
COMPACTNESS IN TOPOLOGICAL TENSOR PRODUCTS AND OPERATOR SPACES
}

\author{
J. R. HOLUB
}

\begin{abstract}
Let $E$ and $F$ be Banach spaces, $E \otimes F$ their algebraic tensor product, and $E \otimes_{\alpha} F$ the completion of $E \otimes F$ with respect to a uniform crossnorm $\alpha \geqq \lambda$ (where $\lambda$ is the "least", and $\gamma$ the greatest, crossnorm). In $\S 2$ we characterize the relatively compact subsets of $E \otimes_{\lambda} F$ as those which, considered as spaces of operators from $E^{*}$ to $F$ and from $F^{*}$ to $E$, take the unit balls in $E^{*}$ and in $F^{*}$ to relatively compact sets in $F$ and $E$, respectively. In $\S 3$ we prove that if $T_{1}: E_{1} \rightarrow E_{2}$ and $T_{2}: F_{1} \rightarrow F_{2}$ are compact operators then $T_{1} \otimes_{\lambda} T_{2}$ and $T_{1} \otimes_{\gamma} T_{2}$ are each compact, and results concerning the problem for an arbitrary crossnorm $\alpha$ are also given. Schatten has characterized $\left(E \otimes_{\alpha} F\right)^{*}$ as a certain space of operators of "finite $\alpha$-norm". In $\S 4$ we show that a space of operators has such a representation if and only if its unit ball is weak operator compact.
\end{abstract}

1. Introduction. Throughout this paper $E$ and $F$ will denote Banach spaces, $E \otimes F$ their algebraic tensor product, and $E \otimes_{\alpha} F$ the completion of $E \otimes F$ with respect to a uniform crossnorm $\alpha \geqq \lambda$ (see [6] for notation and definitions used without other reference). Here $\lambda$ will denote the "least" crossnorm and $\gamma$ the greatest crossnorm [6].

In $\S 2$ we give a characterization of the relatively compact subsets of $E \otimes_{\lambda} F$. The characterization is given in operator terms, regarding $E \otimes_{\lambda} F$ as both a space of compact operators from $E^{*}$ to $F$ and one from $F^{*}$ to $E$.

If $T_{1}: E_{1} \rightarrow E_{2}$ and $T_{2}: F_{1} \rightarrow F_{2}$ are continuous linear operators then the tensor product maps $T_{1} \otimes_{\lambda} T_{2}: E_{1} \otimes_{\lambda} F_{1} \rightarrow E_{2} \otimes_{\lambda} F_{2}$ and $T_{1} \otimes_{\gamma} T_{2}$ : $E_{1} \otimes_{\gamma} F_{1} \rightarrow E_{2} \otimes_{\gamma} F_{2}$ are each continuous. A question of considerable interest and importance in the theory of tensor products is the degree to which properties of the operators $T_{1}$ and $T_{2}$ carry over to the tensor product mappings (for results in this area see [1], [2], [4], and [5]). In $\S 3$ we show that if $T_{1}$ and $T_{2}$ are compact operators then $T_{1} \otimes_{\lambda} T_{2}$ and $T_{1} \otimes_{\gamma} T_{2}$ are also compact. A number of results concerning the more general situation in which $\alpha$ is any crossnorm for which $T_{1} \otimes_{\alpha} T_{2}$ is continuous are also given.

Received by the editors February $8,1972$.

AMS 1970 subject classifications. Primary 46B05, 47D15.

Key words and phrases. Tensor product, space of operators, compact operator, weak operator topology.

(c) American Mathematical Society 1973 
In $\S 4$ we consider the representation of Banach spaces of operators as duals of tensor products. Let $\mathscr{L}(E, F)$ denote the space of all continuous linear operators from $E$ to $F$ with norm given by $\|T\|=\sup _{\|x\|=1}\|T x\|$. A linear subset $A(E, F)$ of $\mathscr{L}(E, F)$ which contains all finite-dimensional operators is called a Banach space of operators (or simply an operator space) if (i) $A(E, F)$ is a $B$-space under some norm which we call the $A$-norm on $A(E, F)$ and denote by $\|\cdot\|_{A}$, and (ii) if $T \in A(E, F)$ then $\|T\|_{A} \geqq\|T\|$, with equality holding for all one-dimensional operators. Schatten has shown that if $\alpha \geqq \lambda$ is a crossnorm on $E \otimes F$ then $\left(E \otimes_{\alpha} F\right)^{*}$ can be identified in a natural way with an operator space $A\left(E, F^{*}\right)$ [6]. In $\S 4$ we show that a given operator space $A\left(E, F^{*}\right)$ can be represented in the form $\left(E \otimes_{\alpha} F\right)^{*}$ for some $\alpha \geqq \lambda$ if and only if the unit ball in $A\left(E, F^{*}\right)$ is compact in the weak operator topology on $A\left(E, F^{*}\right)$.

2. In this section we will characterize the relatively compact subsets of $E \otimes_{\lambda} F$. To state this characterization in operator terms we recall that the space $E \otimes_{\lambda} F$ may be identified (in the obvious way) with the space of all compact operators from $E^{*}$ to $F$ which are continuous in the $w^{*}$-topology on $E^{*}$ and the weak topology on $F$ (of course, the same is true with the roles of $E$ and $F$ reversed) [3]. We will accordingly denote by $T$ an element of $E \otimes_{\lambda} F$, thinking of it as an operator from $E^{*}$ to $F$. Its adjoint $T^{*}: F^{*} \rightarrow E$ is associated with the same element of $E \otimes_{\lambda} F$, of course. Let $U^{0}$ be the unit ball in $E^{*}$ and $V^{0}$ the unit ball in $F^{*}$.

THEOREM 1. A set $A \subset E \otimes_{\lambda} F$ is relatively compact if and only if each of the sets $A\left(U^{0}\right)=\left\{T f \mid T \in A, f \in U^{0}\right\} \subset F$ and $A\left(V^{0}\right)=\left\{T^{*} g \mid T \in A\right.$, $\left.g \in V^{0}\right\} \subset E$ is relatively compact.

Proof. There is a natural embedding of $E \otimes_{\lambda} F$ into $C\left(U^{0} \times V^{0}\right)$ (where $U^{0} \times V^{0}$ has the product of the $w^{*}$-topologies on $U^{0}$ and $V^{0}$ ) obtained by embedding $E \rightarrow C\left(U^{0}\right), F \rightarrow C\left(V^{0}\right)$ and $E \otimes_{\lambda} F \rightarrow C\left(U^{0}\right) \otimes_{\lambda} C\left(V^{0}\right)=$ $C\left(U^{0} \times V^{0}\right)$ [2]. Thus by Ascoli's theorem, to show $A \subset E \otimes_{\lambda} F$ is relatively compact we need only show that as a family of functions in $C\left(U^{0} \times V^{0}\right)$ it is equicontinuous.

Let $\varepsilon>0$ and $\left(f_{0}, g_{0}\right) \in U^{0} \times V^{0}$. Since by assumption the sets $A\left(U^{0}\right)$ and $A\left(V^{0}\right)$ are relatively compact and hence totally bounded there exist $\left(y_{i}\right)_{i=1}^{n} \subset A\left(U^{0}\right)$ and $\left(x_{j}\right)_{j=1}^{m} \subset A\left(V^{0}\right)$ each of which is an $\varepsilon / 6$-net in the respective sets.

If

$$
N\left(f_{0}\right)=\left\{f \in U^{0}||\left\langle f, x_{j}\right\rangle-\left\langle f_{0}, x_{j}\right\rangle \mid<\varepsilon / 6, j=1,2, \cdots, m\right\}
$$

and

$$
N\left(g_{0}\right)=\left\{g \in V^{0}||\left\langle g, y_{i}\right\rangle-\left\langle g_{0}, y_{i}\right\rangle \mid<\varepsilon / 6, i=1,2, \cdots, n\right\}
$$


are $w^{*}$-neighborhoods of $f_{0}$ and $g_{0}$ respectively then for any $(f, g) \in$ $N\left(f_{0}\right) \times N\left(g_{0}\right)$ and any $T \in A$,

$$
\left|\langle T f, g\rangle-\left\langle T f_{0}, g_{0}\right\rangle\right| \leqq\left|\langle T f, g\rangle-\left\langle T f, g_{0}\right\rangle\right|+\left|\left\langle T f, g_{0}\right\rangle-\left\langle T f_{0}, g_{0}\right\rangle\right| .
$$

Now $\left(y_{i}\right)_{i=1}^{n}$ is an $\varepsilon / 6$-net for $A\left(U^{0}\right)$ so there is a $y_{k}$ for which $\left\|T f-y_{k}\right\|<\varepsilon / 6$. Similarly there is an $x_{p}$ for which $\left\|T^{*} g_{0}-x_{p}\right\|<\varepsilon / 6$. Hence

$$
\begin{aligned}
&\left|\langle T f, g\rangle-\left\langle T f, g_{0}\right\rangle\right| \leqq\left|\langle T f, g\rangle-\left\langle y_{k}, g\right\rangle\right|+\left|\left\langle y_{k}, g\right\rangle-\left\langle y_{k}, g_{0}\right\rangle\right| \\
&+\left|\left\langle y_{k}, g_{0}\right\rangle-\left\langle T f, g_{0}\right\rangle\right| \\
&<\varepsilon / 6+\varepsilon / 6+\varepsilon / 6=\varepsilon / 2 .
\end{aligned}
$$

Similarly,

$$
\begin{aligned}
\left|\left\langle T f, g_{0}\right\rangle-\left\langle T f_{0}, g_{0}\right\rangle\right|= & \left|\left\langle f, T^{*} g_{0}\right\rangle-\left\langle f_{0}, T^{*} g_{0}\right\rangle\right| \\
\leqq & \left|\left\langle f, T^{*} g_{0}\right\rangle-\left\langle f, x_{p}\right\rangle\right|+\left|\left\langle f, x_{p}\right\rangle-\left\langle f_{0}, x_{p}\right\rangle\right| \\
& +\left|\left\langle f_{0}, x_{p}\right\rangle-\left\langle f_{0}, T^{*} g_{0}\right\rangle\right| \\
< & \varepsilon / 6+\varepsilon / 6+\varepsilon / 6=\varepsilon / 2 .
\end{aligned}
$$

Combining (2.1), (2.2) and (2.3) we have that if $(f, g) \in N\left(f_{0}\right) \times N\left(g_{0}\right)$ and $T \in A$ then

$$
\left|\langle T f, g\rangle-\left\langle T f_{0}, g_{0}\right\rangle\right|<\varepsilon .
$$

By definition, then, $A$ is an equicontinuous subset of $C\left(U^{0} \times V^{0}\right)$ and is therefore relatively compact.

Conversely, suppose $A \subset E \otimes_{\lambda} F$ is relatively compact. Then given $\varepsilon>0$ there is an $\varepsilon / 2$-net $\left(T_{i}\right)_{i=1}^{n} \subset A$. Since each $T_{i}$ is a compact operator from $E^{*}$ to $F$, the sets $T_{i}\left(U^{0}\right) \subset F$ are each relatively compact and hence totally bounded. Correspondingly, for each $1 \leqq i \leqq n$ there exists a sequence $\left(y_{j}^{(i)}\right)_{j=1}^{m(i)} \subset T_{i}\left(U^{0}\right)$ which is an $\varepsilon / 2$-net for $\bar{T}_{i}\left(\bar{U}^{0}\right)$.

We claim that the set $B=\left\{y_{j}^{(i)} \mid 1 \leqq i \leqq n, 1 \leqq j \leqq m(i)\right\} \subset A\left(U^{0}\right)$ is an $\varepsilon$-net for $A\left(U^{0}\right)$. For, if $T \in A$ then there exists a $1 \leqq k \leqq n$ for which $\left\|T-T_{k}\right\|<\varepsilon / 2$. If $f \in U^{0}$ then $\left\|T_{k} f-T f\right\|<\varepsilon / 2$ and there is a $1 \leqq p \leqq m(k)$ for which $\left\|T_{k} f-y_{p}^{(k)}\right\|<\varepsilon / 2$. It follows that $\left\|T f-y_{p}^{(k)}\right\|<\varepsilon$ and $B$ is an $\varepsilon$-net for $T\left(U^{0}\right)$.

In the same way $A\left(V^{0}\right)$ is relatively compact and the proof is concluded.

Recall that if either $E^{*}$ or $F$ has the approximation property then $E^{*} \otimes_{\lambda} F$ can be identified with the space $K(E, F)$ of all compact operators from $E$ to $F$ [2].

COROLlARY. If either $E^{*}$ or $F$ has the approximation property then a subset $A \subset K(E, F)$ is relatively compact $\Leftrightarrow$ each of the sets $A\left(U^{0}\right)=$ $\{T x \mid T \in A, x \in U=$ unit ball of $E\}$ and $A\left(V^{0}\right)=\left\{T^{*} g \mid T \in A, g \in V^{0}=\right.$ unit ball of $\left.F^{*}\right\}$ are relatively compact. 
3. Let $T_{1}: E_{1} \rightarrow E_{2}$ and $T_{2}: F_{1} \rightarrow F_{2}$ be continuous linear operators. The operator defined on $E_{1} \otimes E_{2}$ by

$$
T_{1} \otimes T_{2}\left(\sum_{i=1}^{n} x_{i} \otimes y_{i}\right)=\sum_{i=1}^{n} T_{1} x_{i} \otimes T_{2} y_{i}
$$

is called the tensor product operator. If $\alpha$ is a $\otimes$-norm (in the terminology of Grothendieck [3]) then $T_{1} \otimes T_{2}$ is continuous and may therefore be extended to the operator $T_{1} \otimes_{\alpha} T_{2}: E_{1} \otimes_{\alpha} F_{1} \rightarrow E_{2} \otimes_{\alpha} F_{2}$. In the study of tensor product spaces it is often necessary to know whether certain properties of the operators $T_{1}$ and $T_{2}$ are inherited by their tensor product. While this is often the case there are numerous exceptions ([2], [4], [5]). In this section we show that compactness of the operators $T_{1}$ and $T_{2}$ carries over to $T_{1} \otimes_{\alpha} T_{2}$ for $\alpha=\lambda$ and $\alpha=\gamma$. Several remarks concerning the problem for arbitrary $\otimes$-norms are also given.

THEOREM 2. Let $T_{1}: E_{1} \rightarrow E_{2}$ and $T_{2}: F_{1} \rightarrow F_{2}$ be compact linear operators. Then $T_{1} \otimes ; T_{2}: E_{1} \otimes_{;} F_{1} \rightarrow E_{2} \otimes_{i} F_{2}$ is compact.

Proof. Let $U^{0}$ and $V^{0}$ denote the unit balls in $E_{2}^{*}$ and $F_{2}^{*}$, respectively. Then, as is well known, we can embed $E_{2}$ in $l^{\infty}\left(U^{0}\right)$ and $F_{2}$ in $l^{\infty}\left(V^{0}\right)$ under the mappings $j_{1}: E_{2} \rightarrow l^{\infty}\left(U^{0}\right)$ and $j_{2}: F_{2} \rightarrow l^{\infty}\left(V^{0}\right)$.

Let $S_{1}=j_{1} \cdot T_{1}$ and $S_{2}=j_{2} \cdot T_{2}$. Then $S_{1} \in K\left(E_{1}, l^{\infty}\left(U^{0}\right)\right)$ and $S_{2} \in$ $K\left(F_{1}, l^{\infty}\left(V^{0}\right)\right)$, so since each of $l^{\infty}\left(U^{0}\right)$ and $l^{\infty}\left(V^{0}\right)$ has the approximation property [2] there exist sequences $\left(P_{n}\right) \subset \mathscr{L}\left(E_{1}, l^{\infty}\left(U^{0}\right)\right)$ and $\left(Q_{n}\right) \subset$ $\mathscr{L}\left(F_{1}, l^{\infty}\left(V^{0}\right)\right)$ of finite-dimensional operators whose norms are uniformly bounded and for which $\left\|S_{1}-P_{n}\right\| \rightarrow{ }^{n} 0$ and $\left\|S_{2}-Q_{n}\right\| \rightarrow{ }^{n} 0$. Then $\left(P_{n} \otimes_{\lambda} Q_{n}\right)$ is a sequence of finite-dimensional operators on $E_{1} \otimes_{\lambda} F_{1}$ into $l^{\infty}\left(U^{0}\right) \otimes_{\lambda}$ $l^{\infty}\left(V^{0}\right)$ for which

$$
\begin{aligned}
\left\|S_{1} \otimes_{\lambda} S_{2}-P_{n} \otimes_{i} Q_{n}\right\| \leqq & \left\|S_{1} \otimes_{\lambda} S_{2}-P_{n} \otimes_{\lambda} S_{2}\right\| \\
& +\left\|P_{n} \otimes_{\lambda} S_{2}-P_{n} \otimes_{i} Q_{n}\right\| \\
\leqq & \left\|S_{2}\right\|\left\|S_{1}-P_{n}\right\|+\left\|P_{n}\right\|\left\|S_{2}-Q_{n}\right\| .
\end{aligned}
$$

Since $\sup _{n}\left\|P_{n}\right\|<+\infty$ this last goes to zero with $n$, showing that $S_{1} \otimes_{\lambda} S_{2}$ can be approximated arbitrarily closely in operator norm by finitedimensional maps and hence is compact.

But $S_{1} \otimes_{i} S_{2}=\left(j_{1} \otimes_{i} j_{2}\right) \cdot\left(T_{1} \otimes_{i} T_{2}\right)$ and it is well known that $j_{1} \otimes_{\lambda} j_{2}$ is an isometry. Therefore $T_{1} \otimes_{i} T_{2}$ is also compact.

REMARK 1. It is clear from the proof of Theorem 2 that the theorem holds for any $\otimes$-norm $\alpha$ for which $j_{1} \otimes_{\alpha} j_{2}$ is an isometry (or even an isomorphism).

REMARK 2. It is also clear from the proof of Theorem 2 that if both $T_{1}$ and $T_{2}$ can be approximated arbitrarily closely by finite-dimensional 
operators then the theorem holds for any $\otimes$-norm. This will be the case, then, if one of $E_{1}$ or $E_{2}$ and one of $F_{1}$ or $F_{2}$ has the approximation property.

THEOREM 3. Let $T_{1}: E_{1} \rightarrow E_{2}$ and $T_{2}: F_{1} \rightarrow F_{2}$ be compact linear operators. Then $T_{1} \otimes_{\gamma} T_{2}: E_{1} \otimes_{\gamma} F_{1} \rightarrow E_{2} \otimes_{\gamma} F_{2}$ is compact.

Proof. It is clear we need only show $T_{1} \otimes T_{2}: E_{1} \otimes F_{1} \rightarrow E_{2} \otimes{ }_{\gamma} F_{2}$ is compact. Let $\left(Z_{k}\right)$ be a $\gamma$-bounded sequence in $E_{1} \otimes F_{1}$, say $Z_{k}=$ $\sum_{i=1}^{n(k)} x_{i}^{(k)} \otimes y_{i}^{(k)}$ for $k=1,2, \cdots$. We want to show that a subsequence of $\left(T Z_{k}\right)$ converges in $E_{2} \otimes_{\gamma} F_{2}$.

To do this, note first that for any $k$ and $p$,

$$
\left\|T_{1} \otimes T_{2}\left(Z_{k}\right)-T_{1} \otimes T_{2}\left(Z_{p}\right)\right\|_{\gamma}=\sup _{\|S\|=1: S \in \mathscr{L}\left(E_{2}, F_{2}^{*}\right)}\left|\left\langle Z_{k}-Z_{p}, T_{2}^{*} S T_{1}\right\rangle\right|
$$

by [6] and the definition of $T_{1} \otimes T_{2}$. Hence we will be able to invoke Ascoli's theorem to obtain the desired result if the set $A=\left\{T_{2}^{*} S T_{1} \mid S \in \mathscr{L}\left(E_{2}, F_{2}^{*}\right)\right.$ with $\|S\| \leqq 1\}$ is relatively compact in $\mathscr{L}\left(E_{1}, F_{1}^{*}\right)=\left(E_{1} \otimes_{\gamma} F_{1}\right)^{*}$ (since $\left(Z_{k}\right)$ is an equicontinuous subset of $C(A)$ ).

Let $\left\{T_{2}^{*} S_{n} T_{1}\right\}$ be a sequence in $A$. For any $n, m$,

$$
\begin{aligned}
\left\|T_{2}^{*} S_{n} T_{1}-T_{2}^{*} S_{m} T_{1}\right\| & =\left\|T_{2}^{*}\left(S_{n}-S_{m}\right) T_{1}\right\| \\
& =\sup _{\|x\|=1, x \in E_{1}:\|y\|=1, y \in F_{1}}\left|\left\langle T_{2}^{*}\left(S_{n}-S_{m}\right) T_{1} x, y\right\rangle\right| \\
& =\sup _{\|x\|=1, x \in E_{1}:\|y\|=1 . y \in F_{1}}\left|\left\langle S_{n}-S_{m} T_{1} x, T_{2} y\right\rangle\right| .
\end{aligned}
$$

Again, since $\left(S_{n}\right)$ is an equicontinuous subset of $\left(E_{2} \otimes_{\gamma} F_{2}\right)^{*}$, our theorem will be proved if the set $T_{1}(U) \otimes T_{2}(V)=\left\{T_{1} x \otimes T_{2} y\|x\| \leqq 1,\|y\| \leqq 1\right\}$ is relatively compact in $E_{2} \otimes_{\gamma} F_{2}$. But this is immediate since if $\left(T_{1} x_{n} \otimes T_{2} y_{n}\right)$ is a sequence in $T_{1}(U) \otimes T_{2}(V)$, then by virtue of the fact that each of $T_{1}$ and $T_{2}$ is compact there exist subsequences $\left(T_{1} x_{n_{k}}\right)$ and $\left(T_{2} y_{n_{k}}\right)$ which converge in $E_{2}$ and $F_{2}$, respectively, and hence for which $\left(T_{1} x_{n_{k}} \otimes T_{2} y_{n_{k}}\right)$ also converges. The theorem is proved.

In view of Remark 2 following Theorem 2 it is, of course, very likely that the tensor product $T_{1} \otimes_{\alpha} T_{2}$ of compact operators is compact for every $\otimes$-norm $\alpha$ (we continue to require $\alpha$ to be $\mathrm{a} \otimes$-norm only to insure that $T_{1} \otimes_{\alpha} T_{2}$ is continuous). The next theorem shows that the problem may be reduced to that of showing that one certain type of tensor product map is compact.

Let $E$ and $F$ be Banach spaces and $\left(f_{n}\right) \subset E^{*},\left(g_{n}\right) \subset F^{*}$ sequences for which $\left\|f_{n}\right\| \rightarrow 0,\left\|g_{n}\right\| \rightarrow 0$. Define the mappings $T \in K\left(E, c_{0}\right)$ and $S \in$ $K\left(F, c_{0}\right)$ by

$$
T x=\left(\left\langle f_{n}, x\right\rangle\right), \quad S y=\left(\left\langle g_{n}, y\right\rangle\right)
$$


If $X=$ closure of the range of $T$ and $Y=$ closure of the range of $S$ then $T \in K(E, X)$ and $S \in K(F, Y)$.

THEOREM 4. Let $\alpha$ be a $\otimes$-norm such that whenever $T \in K(E, X)$ and $S \in K(F, Y)$ are as above then $T \otimes_{\alpha} S: E \otimes_{\alpha} F \rightarrow X \otimes_{\alpha} Y$ is a compact operator. Then if $T_{1}: E_{1} \rightarrow E_{2}$ and $T_{2}: F_{1} \rightarrow F_{2}$ are compact operators the tensor product $T_{1} \otimes_{\alpha} T_{2}$ is also compact.

Proof. It is known that since $T_{1}$ and $T_{2}$ are compact there exist sequences $\left(f_{n}\right) \subset E_{1}^{*}$ and $\left(g_{n}\right) \subset F_{1}^{*}$ such that $\left\|f_{n}\right\| \rightarrow 0,\left\|g_{n}\right\| \rightarrow 0$ and $\left\|T_{1} x\right\| \leqq \sup _{n}\left|\left\langle f_{n}, x\right\rangle\right|$ for all $x \in E_{1},\left\|T_{2} y\right\| \leqq \sup _{n}\left|\left\langle g_{n}, y\right\rangle\right|$ for all $y \in F_{1}$ [7].

Thus $T_{1}$ may be factored as $T_{1}=P_{2} \cdot P_{1}$ where $P_{1}(x)=\left(\left\langle f_{n}, x\right\rangle\right) \in X \subset C_{0}$ for all $x \in E_{1}$ and $P_{2}: X \rightarrow E_{2}$ is defined by $P_{2}\left(\left\langle f_{n}, x\right\rangle\right)=T_{1} x$. Similarly $T_{2}$ can be factored as $T_{2}=Q_{2} \cdot Q_{1}$ where $Q_{1}(y)=\left(\left\langle g_{n}, y\right\rangle\right) \in Y \subset C_{0}$ for $y \in F_{1}$ and $Q_{2}: Y \rightarrow F_{2}$ is defined by $Q_{2}\left(\left\langle g_{n}, y\right\rangle\right)=T_{2} y$.

Since $T_{1} \otimes_{\alpha} T_{2}=\left(P_{2} \otimes_{\alpha} Q_{2}\right) \cdot\left(P_{1} \otimes_{\alpha} Q_{1}\right)$, where $P_{1} \otimes_{\alpha} Q_{1}: E_{1} \otimes_{\alpha} F_{1} \rightarrow$ $X \otimes_{\alpha} Y$ and $P_{2} \otimes_{\alpha} Q_{2}: X \otimes_{\alpha} Y \rightarrow E_{2} \otimes_{\alpha} F_{2}$, and by assumption $P_{1} \otimes_{\alpha} Q_{2}$ is compact, we see that $T_{1} \otimes_{\alpha} T_{2}$ is also compact.

REMARK 3. If one could show that the sets $X$ and $Y$ occurring in the above theorem have the approximation property then Theorem 4 together with Remark 2 would prove the result for all $\alpha$.

4. If $E$ and $F$ are Banach spaces and $\alpha$ a crossnorm on $E \otimes F$ then according to results of Schatten the space $\left(E \otimes_{\alpha} F\right)^{*}$ can be identified with the Banach space $A_{\alpha}\left(E, F^{*}\right)$ of all operators from $E$ to $F^{*}$ of "finite $\alpha$-norm" [6]. In this section we study the converse problem, that of determining when a given space of operators $A\left(E, F^{*}\right)$ is (under the identification given by Schatten) a space $A_{\alpha}\left(E, F^{*}\right)=\left(E \otimes_{\alpha} F\right)^{*}$ for some crossnorm $\alpha$.

The definition of a Banach space of operators $A\left(E, F^{*}\right)$ was given in $\S 1$. By the weak operator topology on $A\left(E, F^{*}\right)$ we mean the topology of pointwise convergence of nets in $A\left(E, F^{*}\right)$ on the set $E \times F$ (or, equivalently, on $\left.E \otimes F \subset A\left(E, F^{*}\right)^{*}\right)$.

We begin with a simple lemma. We emphasize that the embedding mentioned in the lemma (and denoted by the inclusion symbol) refers, as do similar embeddings throughout this section, to a very special embedding (namely, that of Schatten) which is explicitly defined in the proof of the lemma.

Lemma. If $A\left(E, F^{*}\right)$ is an operator space and $\alpha$ is defined on $E \otimes F$ by

$$
\alpha\left(\sum_{i=1}^{n} x_{i} \otimes y_{i}\right)=\sup _{\|T\|_{A=1}}\left|\sum_{i=1}^{n}\left\langle T x_{i}, y_{i}\right\rangle\right|,
$$

then $\alpha$ is a crossnorm and $E \otimes_{\alpha} F \subset A\left(E, F^{*}\right)^{*}$. 
Proof. If $\sum_{i=1}^{n} x_{i} \otimes y_{i} \in E \otimes F$ then for $T \in A\left(E, F^{*}\right)$ the equation $\left\langle T, \sum_{i=1}^{n} x_{i} \otimes y_{i}\right\rangle=\sum_{i=1}^{n}\left\langle T x_{i}, y_{i}\right\rangle$ identifies the tensor $\sum_{i=1}^{n} x_{i} \otimes y_{i}$ with a linear functional on $A\left(E, F^{*}\right)$. If $\left(T_{m}\right)$ is a sequence in $A\left(E, F^{*}\right)$ converging in $A$-norm to zero them $\left(T_{m}\right)$ also converges to zero in operator norm (since $\|T\|_{A} \geqq\|T\|$ for $T \in A\left(E, F^{*}\right)$ ), implying $\sum_{i=1}^{n}\left\langle T_{m} x_{i}, y_{i}\right\rangle \rightarrow^{m} 0$ and the functional $\sum_{i=1}^{n} x_{i} \otimes y_{i}$ is continuous on $A\left(E, F^{*}\right)$. Therefore we can identify in a canonical fashion the algebraic tensor product $E \otimes F$ with a linear subspace of $A\left(E, F^{*}\right)^{*}$. If we define

$$
\alpha\left(\sum_{i=1}^{n} x_{i} \otimes y_{i}\right)=\sup _{\|T\| A=1}\left|\sum_{i=1}^{n}\left\langle T x_{i}, y_{i}\right\rangle\right|
$$

then $\alpha$ is simply the norm induced on $E \otimes F$ considered as a subspace of $A\left(E, F^{*}\right)^{*}$ and so the embedding $E \otimes_{\alpha} F \rightarrow A\left(E, F^{*}\right)^{*}$ is an isometry.

Moreover if $x \otimes y \in E \otimes F$ then

$$
\alpha(x \otimes y)=\sup _{\|T\|_{A}=1}\langle T x, y\rangle \leqq \sup _{\|T\|=1}\langle T x, y\rangle \leqq\|x\|\|y\| .
$$

Conversely,

$$
\alpha(x \otimes y) \geqq \sup _{\|f\|=\|g\|=1}\langle x \otimes y, f \otimes g\rangle=\|x\|\|y\|
$$

since by assumption $A\left(E, F^{*}\right)$ contains all finite-dimensional operators and the $A$-norm of the one-dimensional operator $f \otimes g$ is equal to $\|f \otimes g\|=$ $\|f\| \cdot\|g\|$. It follows that $\alpha$ is a crossnorm and the lemma is proved.

REMARK. Though we will not need the result in what follows, the crossnorm $\alpha$ defined above is $\geqq \lambda$. To see this note that

$$
\begin{aligned}
\alpha\left(\sum_{i=1}^{n} x_{i} \otimes y_{i}\right) & =\sup _{\|T\| .=1}\left\langle\sum_{i=1}^{n} x_{i} \otimes y_{i}, T\right\rangle \\
& \geqq \sup _{\|f\|=\|s\|=1}\left\langle\sum_{i=1}^{n} x_{i} \otimes y_{i}, f \otimes g\right\rangle
\end{aligned}
$$

(as in the proof of the lemma)

$$
=\left\|\sum_{i=1}^{n} x_{i} \otimes y_{i}\right\|_{\lambda} .
$$

Using this lemma we now prove the converse to Schatten's theorem.

THEOREM 5. Let $A\left(E, F^{*}\right)$ be an operator space. Then there is a crossnorm $\alpha$ for which $A\left(E, F^{*}\right)=\left(E \otimes_{\alpha} F\right)^{*}$ if and only if whenever $T \in \mathscr{L}\left(E, F^{*}\right)$ is the weak operator limit of a net $\left(T_{\gamma}\right)$ in $A\left(E, F^{*}\right)$ for which $\sup _{\gamma}\left\|T_{y}\right\|_{A}<$ $+\infty$ then $T \in A\left(E, F^{*}\right)$ and $\|T\|_{A} \leqq \sup _{\gamma}\left\|T_{\gamma}\right\|_{A}$. 
Proof. Suppose $A\left(E, F^{*}\right)=A_{\alpha}\left(E, F^{*}\right)=\left(E \otimes_{\alpha} F\right)^{*}$ for some crossnorm $\alpha$ and $T \in \mathscr{L}\left(E, F^{*}\right)$ is the weak operator limit of the net $\left(T_{\gamma}\right)$ in $A\left(E, F^{*}\right)$ for which $\sup _{\gamma}\left\|T_{\gamma}\right\|_{A}=M<+\infty$.

If $\sum_{i=1}^{n} x_{i} \otimes y_{i} \in E \otimes_{\alpha} F$ and $\varepsilon>0$ then there is a $\gamma_{0}$ for which

Hence

$$
\left|\sum_{i=1}^{n}\left\langle T x_{i}, y_{i}\right\rangle-\sum_{i=1}^{n}\left\langle T_{\gamma_{0}} x_{i}, y_{i}\right\rangle\right|<\varepsilon .
$$

$$
\begin{aligned}
\left|\sum_{i=1}^{n}\left\langle T x_{i}, y_{i}\right\rangle\right| & <\left|\sum_{i=1}^{n}\left\langle T_{y_{0}} x_{i}, y_{i}\right\rangle\right|+\varepsilon \\
& \leqq\left\|T_{\gamma_{0}}\right\|_{A}\left\|\sum_{i=1}^{n} x_{i} \otimes y_{i}\right\|_{\alpha}+\varepsilon \leqq M\left\|\sum_{i=1}^{n} x_{i} \otimes y_{i}\right\|_{\alpha}+\varepsilon .
\end{aligned}
$$

Since $\varepsilon>0$ was arbitrary we see that $T$ is a continuous linear functional on $E \otimes_{\alpha} F$ and is therefore by Schatten's theorem an element of $A_{\alpha}\left(E, F^{*}\right)=$ $A\left(E, F^{*}\right)$. Moreover, $\|T\|_{A} \leqq M=\sup _{y}\left\|T_{y}\right\|_{A}$.

Conversely, suppose the operator space $A\left(E, F^{*}\right)$ has the stated property. Define $\alpha$ on $E \otimes F$ as in the preceding lemma. Then according to the lemma the canonical mapping $S: E \otimes_{\alpha} F \rightarrow A\left(E, F^{*}\right)^{*}$ described in the proof is an isometric isomorphism.

Let $T \in\left(E \otimes_{\alpha} F\right)^{*}=A_{\alpha}\left(E, F^{*}\right)$. By the Hahn-Banach theorem, $T$ extends to a functional $\tilde{T}$ in $A\left(E, F^{*}\right)^{* *}$ with $\|T\|_{A_{\alpha}\left(E, F^{*}\right)}=\|\tilde{T}\|_{A\left(E, F^{*}\right)^{* *}}$. Since the unit ball in $A\left(E, F^{*}\right)$ is weak*-dense in the unit ball of $A\left(E, F^{*}\right)^{* *}$ there exists a net $\left(S_{\gamma}\right)$ in $A\left(E, F^{*}\right)$ for which $\sup _{\gamma}\left\|S_{\gamma}\right\|_{A} \leqq\|\tilde{T}\|$ and $\left\langle S_{\gamma}, x \otimes y\right\rangle \rightarrow\langle\tilde{T}, x \otimes y\rangle=\langle T x, y\rangle$ for all $x \otimes y \in E \otimes F \subset A\left(E, F^{*}\right)^{*}$. It follows, then, that we actually have $\left(S_{\gamma}\right)$ weak operator convergent to $T$. By hypothesis, then, $T \in A_{\alpha}\left(E, F^{*}\right)$ and $\|T\|_{A} \leqq \sup _{\gamma}\left\|S_{\gamma}\right\| \leqq\|T\|_{A_{\alpha}\left(E, F^{*}\right)}$.

Thus we have shown that if $T \in A_{\alpha}\left(E, F^{*}\right)$ then $T \in A\left(E, F^{*}\right)$. The reverse inclusion is trivial since if $T \in A\left(E, F^{*}\right)$ then $T$ defines a continuous linear functional on $A\left(E, F^{*}\right)^{*}$ and hence also on $E \otimes_{\alpha} F \subset A\left(E, F^{*}\right)^{*}$. By Schatten's theorem $T \in A_{\alpha}\left(E, F^{*}\right)$ and $\|T\|_{A_{\alpha}\left(E, F^{*}\right)} \leqq T \|_{-A}$. It follows that the spaces $A\left(E, F^{*}\right)$ and $A_{\alpha}\left(E, F^{*}\right)$ are isometrically isomorphic.

A more useful version of Theorem 5 is

CoROllary. Let $A\left(E, F^{*}\right)$ be an operator space. Then there is a crossnorm $\alpha \geqq \lambda$ such that $A\left(E, F^{*}\right)=\left(E \otimes_{\alpha} F\right)^{*}$ if and only if the unit ball in $A\left(E, F^{*}\right)$ is compact in the weak operator topology.

Proof. If $A\left(E, F^{*}\right)=\left(E \otimes_{\alpha} F\right)^{*}$ then the unit ball in $A\left(E, F^{*}\right)$ is compact in the $\sigma\left(A,\left(E, F^{*}\right), E \otimes_{\alpha} F\right)$-topology (by Alaoglu's theorem) and hence certainly weak operator compact since all $x \otimes y$ for $x \in E$, $y \in F$ are in $E \otimes_{\alpha} F$. 
Conversely, suppose the unit ball in $A\left(E, F^{*}\right)$ is compact in the weak operator topology and $T \in \mathscr{L}\left(E, F^{*}\right)$ is the weak operator limit of the bounded net $\left(T_{\gamma}\right)$ in $A\left(E, F^{*}\right)$. Then by compactness of the unit ball there is an operator $S$ in the ball of radius $r=\sup _{\gamma}\left\|T_{\gamma}\right\|_{A}$ in $A\left(E, F^{*}\right)$ which is a weak operator cluster point of $\left(T_{\gamma}\right)$. But since $\left(T_{\gamma}\right)$ converges to $T$ in weak operator topology it must be that $S=T$ (the weak operator topology is Hausdorff), and hence, by Theorem 5, $A\left(E, F^{*}\right)=\left(E \otimes_{\alpha} F\right)^{*}$ for the crossnorm $\alpha$ constructed in that theorem.

\section{REFERENCES}

1. A. Brown and C. Pearcy, Spectra of tensor products of operators, Proc. Amer. Math. Soc. 17 (1966), 162-166. MR 32 \#6218.

2. A. Grothendieck, Produits tensoriels topologiques et espaces nucléaires, Mem. Amer. Math. Soc. No. 16 (1955). MR 17, 763.

3. —- Résumé de la théorie métrique des produits tensoriels topologiques, Bol. Soc. Mat. São Paulo 8 (1953), 1-79. MR 20 \#1194.

4. J. Holub, Tensor product mappings, Math. Ann. 188 (1970), 1-12.

5. T. Ichinose, On the spectra of tensor products of linear operators in Banach spaces, J. Reine Angew. Math. 244 (1970), 119-153. MR 43 \#3828.

6. R. Schatten, A theory of cross-spaces, Ann. of Math. Studies, no. 26, Princeton Univ. Press, Princeton, N.J., 1950. MR 12, 186.

7. T. Terzioglu, A characterization of compact linear mappings, Arch. Math. 22 (1971), 76-78.

Department of Mathematics, Virginia Polytechnic instinute and State UNIVERSITY, BLACKSBURG, VIRGINIA 24061 